\title{
Modelos de geração de viagem para pólos geradores de viagens de cargas
}

\author{
Cristiane Duarte Ribeiro de Souza'; Suellem Deodoro Silva²; \\ Márcio de Almeida D'agosto ${ }^{3}$
}

\begin{abstract}
Resumo: A implantação de um empreendimento que tenha potencial de produzir ou atrair uma quantidade significativa de viagens de cargas (PGV Carga) pode contribuir para o agravamento dos problemas de tráfego já existentes ou ocasionar problemas não previstos em uma região. Conhecer o volume de viagens geradas e seus modelos de previsão pode ajudar a minimizar tais problemas. Este trabalho tem por objetivo apresentar o perfil dos modelos de geração de viagens de carga que possam ser utilizados para subsidiar os estudos sobre PGV Carga. Foi realizada uma revisão bibliográfica nacional e internacional ( 25 estudos). Verificou-se que predomina o uso de taxas e de regressões lineares simples e múltiplas, nas quais se obtêm coeficientes de determinação $\left(\mathrm{R}^{2}\right)$ iguais ou maiores que 0,7 .
\end{abstract}

Abstract: The deployment of an enterprise that has the potential to produce or attract a significant amount of freight travel, may contribute to the worsening traffic problems that already exist or cause unanticipated problems in a region. Knowing the volume of trips generated and their forecasting models can help minimize such problems. This paper aims to present a profile of freight trip generation models that can be used to subsidize research on such enterprises. We performed a national and international literature review ( 25 studies). It was found the predominant use of taxes and simple and multiple linear regressions, where we obtain coefficients of determination $\left(\mathrm{R}^{2}\right)$ equal to or greater than 0.7 .

\section{INTRODUÇÃO}

As diferentes atividades sociais e econômicas que são desenvolvidas nas áreas urbanas geram a necessidade de movimentação de pessoas e de carga. O transporte de carga é uma atividade de destaque atualmente por sua participação no produto interno bruto dos países e pela crescente influência que a transferência e a distribuição de bens têm no desempenho de todos os setores econômicos (Crainc e Laporte, 1997).

É preciso analisar os problemas que podem ser gerados pelo transporte de carga, principalmente, em área urbana. A movimentação de carga e o serviço realizado por caminhões nessas áreas, aliada às necessidades de carga e descarga, podem agravar os problemas de tráfego já existentes na cidade, impactando aquele que contrata e efetua o transporte, o cidadão e o poder público (Facchini, 2006). Neste contexto, a implantação de um empreendimento que tenha potencial de produzir ou atrair uma quantidade significativa de viagens de cargas (PGV Carga) pode contribuir pa-

\footnotetext{
${ }^{1}$ Cristiane Duarte Ribeiro de Souza, Programa de Engenharia de Transportes, Instituto Alberto Luiz Coimbra de Pós-Graduação e Pesquisa de Engenharia, Universidade Federal do Rio de Janeiro, Rio de Janeiro, RJ, Brasil. (e-mail: cristiane@pet.coppe.ufr..br).

${ }^{2}$ Suellem Deodoro Silva, Programa de Engenharia de Transportes, Instituto Alberto Luiz Coimbra de Pós-Graduação e Pesquisa de Engenharia, Universidade Federal do Rio de Janeiro, Rio de Janeiro, RJ, Brasil. (e-mail: suellem_04@pet.coppe.ufrj.br).

${ }^{3}$ Márcio de Almeida D'agosto, Programa de Engenharia de Transportes, Instituto Alberto Luiz Coimbra de Pós-Graduação e Pesquisa de Engenharia, Universidade Federal do Rio de Janeiro, Rio de Janeiro, RJ, Brasil. (e-mail: dagosto@pet.coppe.ufrj.br).
}

Manuscrito recebido em 7/12/2009 e aprovado para publicação em 5/2/2010. Este artigo é parte de TRANSPORTES, volume XVIII, número 1, março de 2010. ISSN: 2237-1346 (online). ra o agravamento dos problemas de tráfego já existentes ou ocasionar problemas não previstos.

Este trabalho tem por objetivo apresentar o perfil dos modelos de geração de viagens de carga que podem vir a ser utilizados para subsidiar os estudos sobre PGV Carga. O trabalho está dividido em 5 itens. O $1^{\circ}$ apresenta uma breve introdução sobre o tema estudado. $\mathrm{O} 2^{\circ}$ trata da metodologia utilizada para a realização do trabalho. $\mathrm{O} 3^{\circ}$ item apresenta os resultados de uma revisão bibliográfica nacional e internacional sobre modelos de geração de viagens aplicados à PGV Carga, sendo seguido por uma discussão desses resultados (item 4). No item 5, apresentam-se as considerações finais e as sugestões para futuros trabalhos.

\section{METODOLOGIA}

O presente trabalho foi desenvolvido conforme fluxograma apresentado na Figura 1.

Desta forma, visando identificar o perfil dos modelos de geração de viagens utilizados em estudos de PGV Carga, realizou-se uma revisão bibliográfica com abrangência nacional e internacional que considerou 25 trabalhos, dentre eles 4 nacionais e 21 internacionais, com a distribuição geográfica apresentada na Figura 2 (a) e distribuídos no período de 1974 a 2009, verificando-se leve concentração (12\%) de estudos no ano de 2002. Como fontes de pesquisa utilizaram-se livros, periódicos internacionais, teses, dissertações, artigos publicados em congressos nacionais, manuais e relatórios técnicos (Figura 2 (b)). A revisão bibliográfica preliminar teve por objetivo identificar autores e conceitos relacionados ao tema, verificando as particularidades existentes na modelagem da de- 


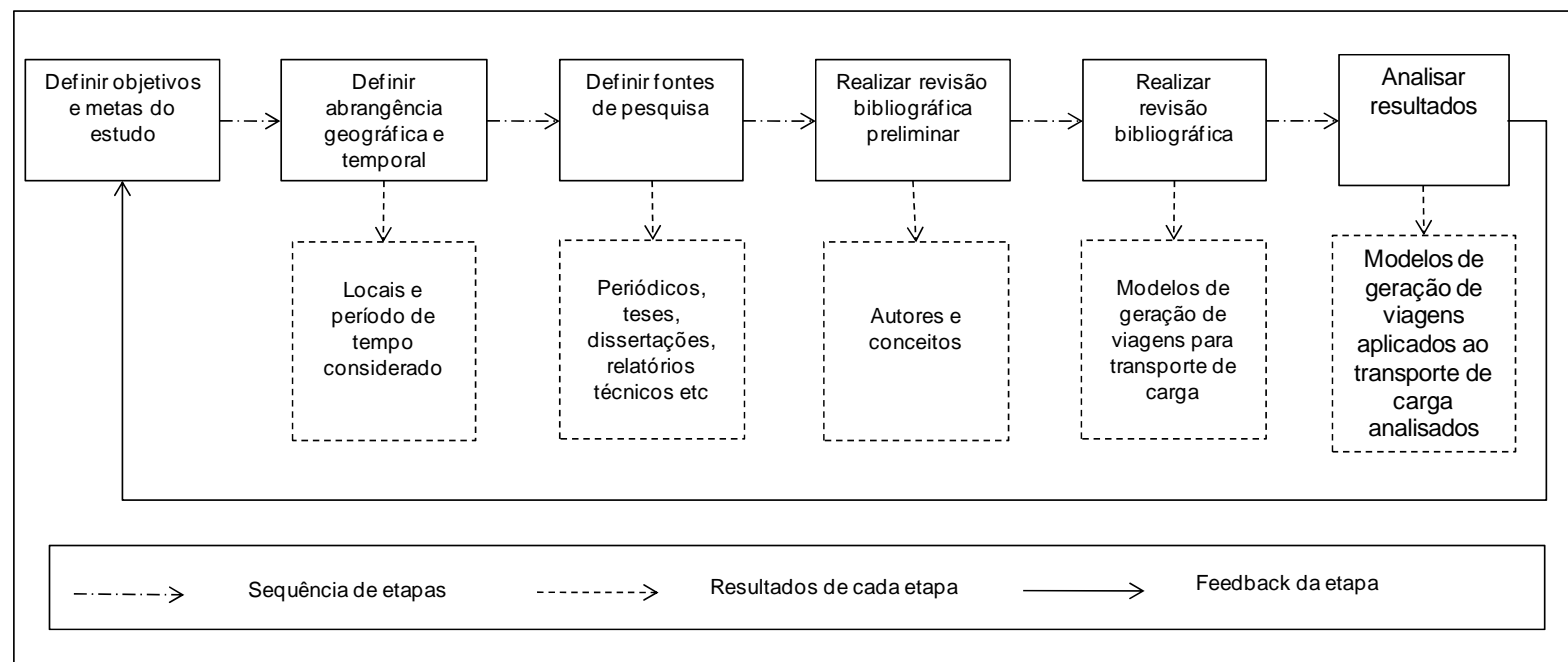

Figura 1. Metodologia adotada para elaboração do estudo em questão

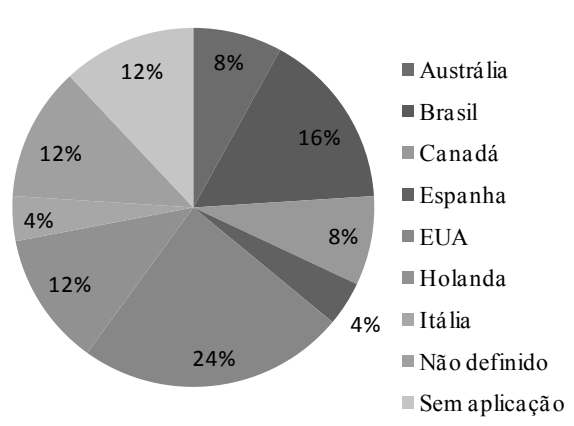

(a) Abrangência do estudo

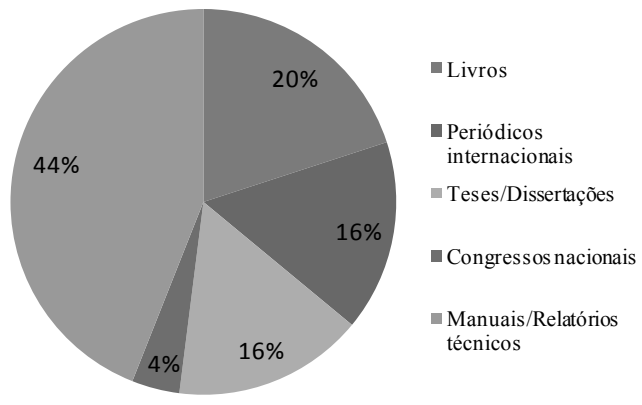

(b) Fontes pesquisadas

Nota: Entende-se por "Não definido" o estudo que não apresentou local definido e por "Sem aplicação" os estudos que não apresentaram aplicação dos modelos

Figura 2. Distribuição geográfica e temporal dos estudos analisados

manda de transporte de carga e as diferentes abordagens utilizadas.

A complexidade de se modelar a demanda de transporte de carga surge da necessidade de se considerar a existência de diversas dimensões (diferentes tipos de carga, volumes, pesos e volume de viagens) sob o controle de diferentes tomadores de decisão (embarcadores, transportadores - motoristas e despachantes) que interagem em um ambiente dinâmico. Desse modo, ao se modelar a demanda por viagens de carga, verificam-se duas principais abordagens derivadas do modelo de quatro etapas (geração de viagens, distribu-

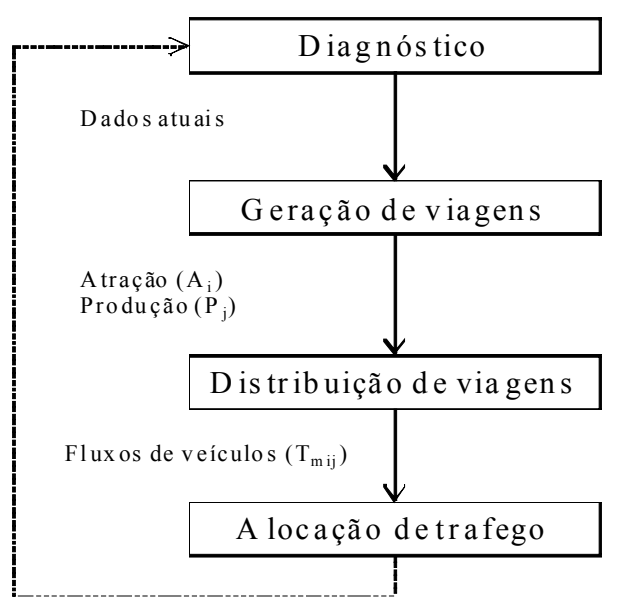

Flux os de veículos $\left(\mathrm{T}_{\mathrm{myij}}\right)$

(a) Abordagem com base em volume de viagens

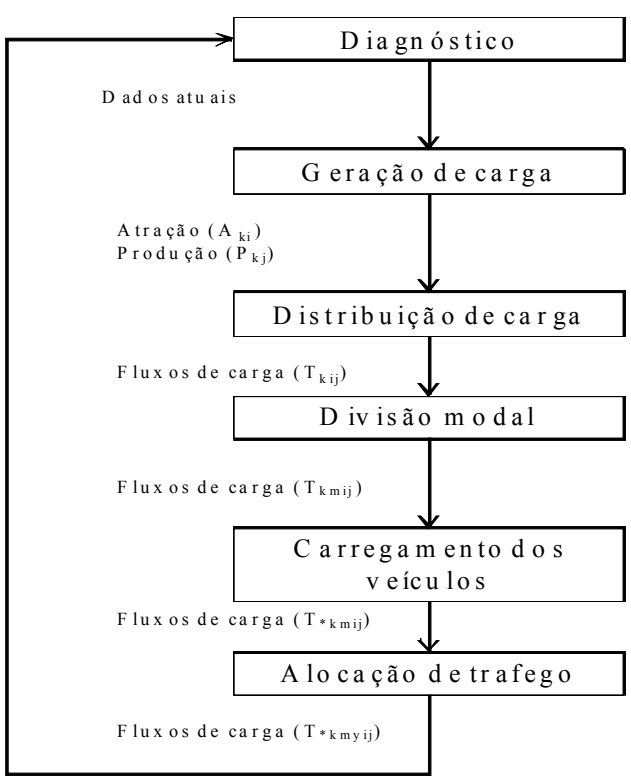

(b) Abordagem com base em volume de carga

Nota: $i$ - origem, $j$ - destino, $m$ - modo de transporte, $k$ - tipo de carga, $y$ - rota

Figura 3. Modelos de quatro etapas adaptado para carga 
ição de viagens, divisão modal e alocação de tráfego): o uso de modelos baseados em volume de viagens e o uso de modelos baseados em volume de carga (Holguín-Veras e Thorson, 2000 e Ogden, 1977).

Os modelos baseados em volume de viagens, como o apresentado na Figura 3 (a), têm seu foco no fluxo de veículos (origem i e destino j). Esse modelo pressupõe que a seleção do modo de transporte $(\mathrm{m})$ já foi realizada e, portanto, não se faz necessário efetuar a etapa de divisão modal, passando-se da distribuição de viagens para a alocação de tráfego (y).

Já os modelos baseados em volume de carga (k), como o apresentado na Figura 3 (b), têm o foco na quantidade de carga transportada medida em toneladas, ou em qualquer outra unidade de peso. Esse modelo permite captar com maior precisão os mecanismos que direcionam a movimentação das cargas, função de atributos, como forma e peso por unidade.

\section{MODELOS DE GERAÇÃO DE VIAGENS - EXPERIÊNCIAS NACIONAIS E INTERNACIONAIS}

Este item apresenta os resultados da pesquisa realizada com o intuito de identificar qual o perfil dos modelos de geração de demanda de viagens de carga aplicados a estudos de PGV Carga. Os trabalhos nacionais que consideram modelos de geração de demanda de viagens de carga aplicados a estudos de PGV Carga encontram-se no Quadro 1. Já os trabalhos internacionais são apresentados nos Quadros 2-A, 2-B, 2-C, 2-D e 2-E. Além dos estudos organizados nos Quadros $1 \mathrm{e}$ 2, foram analisados mais 3 estudos: Munuzuni et al. (2009), Nuzzolo et al. (2008) e Morlok (1978). Como esses estudos não apresentaram aplicação, optou-se por mencioná-los apenas durante a discussão dos resultados (item 4).

\section{DISCUSSÃO DOS RESULTADOS}

No Quadro 1 é possível verificar as experiências nacionais analisadas. O trabalho de Gasparini (2008) realizou um estudo considerando shopping centers e supermercados. Para a elaboração do modelo de geração de viagens, o autor utilizou uma modelagem baseada em viagens de veículos e aplicou regressão linear simples (não apresentadas) e múltipla. Como variáveis dependentes, foram consideradas: o volume total de viagens por veículos que são atraídos ao PGV (caminhões, vans/furgões, carros baú, picapes, carros forte, carros dos correios e motos) ou apenas os caminhões.

Para o caso dos shopping centers, foram feitas observações em 2 períodos de uma semana, identificando-se os horários de pico e entre-pico ou todo o período para um total de 7 empreendimentos. Já para o caso dos supermercados, foram realizadas observações por
1 período de uma semana para um total de 21 empreendimentos. Entretanto, em virtude da similaridade de forma observada entre as equações elaboradas para o período de pico e entre-pico com as equações elaboradas para o período total, optou-se por apresentar (Quadro 1) apenas as equações que representam o volume de viagens para o período total.

Verifica-se que para o estudo realizado em shoppings centers foram utilizadas como variáveis independentes a área construída, o volume médio de clientes e a área bruta locável. Já para o estudo em supermercados, foram utilizadas as variáveis área de vendas, número de vagas no estacionamento, volume médio de clientes e números de empregados.

O modelo de geração de viagens elaborado por Silva e Waisman (2007) teve como base uma pesquisa estruturada por meio de questionário e entrevista realizada na cidade de São Paulo em 30 bares e restaurantes. Os autores também empregam uma modelagem baseada em viagens de veículos, utilizando as variáveis: área construída e número de empregados. Nos 30 estabelecimentos pesquisados, a área variou de $60 \mathrm{a}$ $500 \mathrm{~m}^{2}$ e de 2 a 17 empregados por estabelecimento.

O estudo de Melo (2002) foi desenvolvido na cidade do Rio de Janeiro com o intuito de modelar a demanda por transporte de carga urbana atraída por lojas comerciais e produzida por empresas de transporte. A autora utiliza uma modelagem baseada em viagens de veículos e aplica regressão linear. Para os modelos relacionados ao setor de lojas comerciais, a autora utiliza regressão linear simples. Já para os modelos relacionados ao setor de transporte, é utilizada regressão linear múltipla. Analisando o Quadro 1, verifica-se que apenas 2 equações apresentam coeficiente de determinação $\left(\mathrm{R}^{2}\right)$ maior que 0,7 , considerado como bom neste trabalho.

Marra (1999) apresenta um estudo realizado no estado de São Paulo, em que se utilizou uma modelagem baseada em volume de carga. Inicialmente, o autor utilizou regressão linear simples e ajuste exponencial para obter o volume de carga com base em 3 variáveis independentes distintas (área construída, número de empregados/moradores e rendimento médio per capita). O autor elaborou também uma equação para cada um dos bairros estudados, utilizando regressão linear simples e exponencial. Outros ajustes foram apresentados, porém, devido à baixa aderência dos dados a essas outras equações, optou-se por não apresentá-las neste estudo. Os maiores coeficientes de determinação foram obtidos para as regressões lineares e ajuste exponencial. 


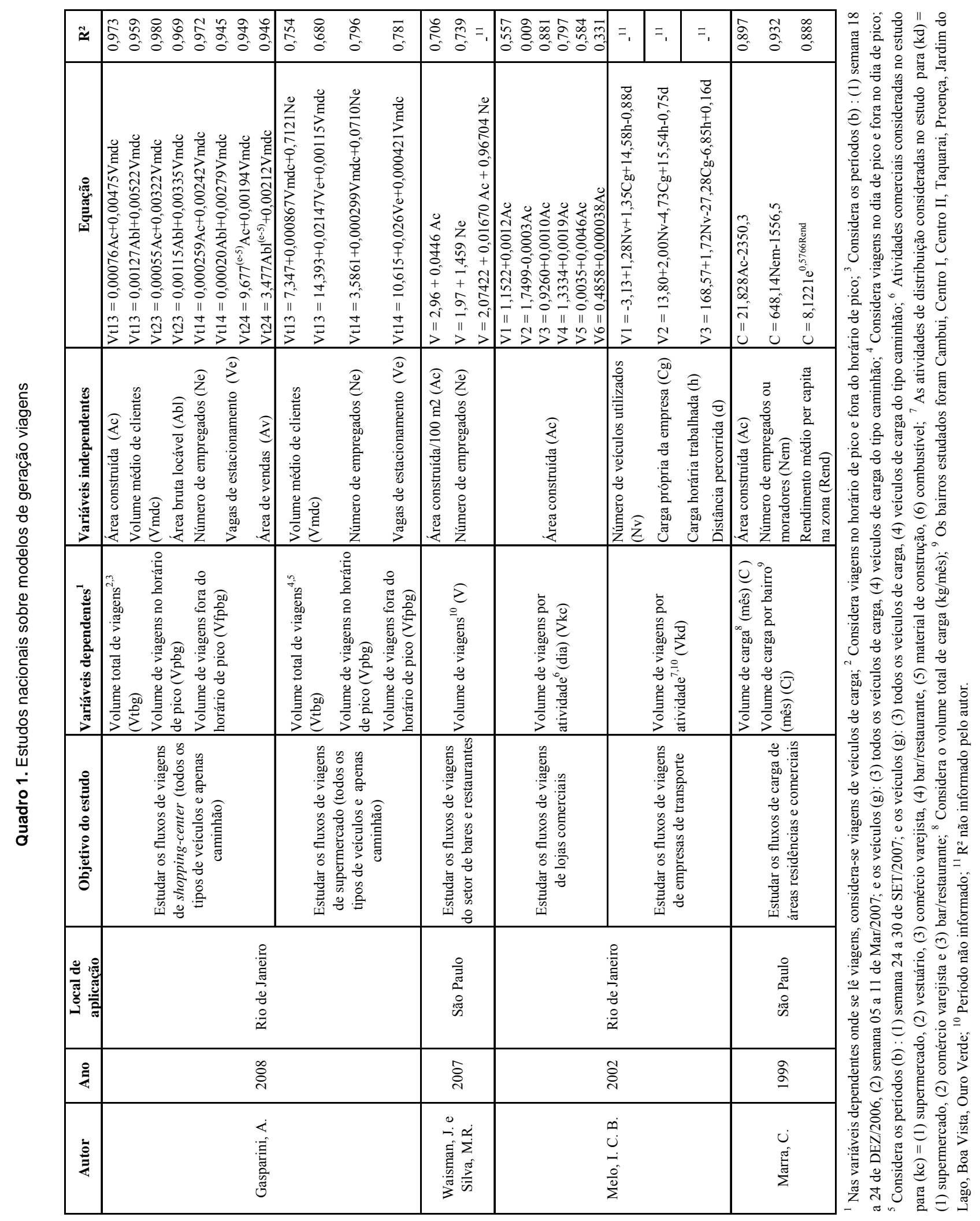


Os Quadros 2-A, 2-B, 2-C, 2-D e 2-E apresentam as experiências internacionais analisadas. Os modelos de geração de viagens para caminhões médios e para caminhões pesados desenvolvidos por Allen (2002) e apresentados no Quadro 2-A baseiam-se em uma modelagem de viagens de carga, considerando como variáveis independentes: o número de empregos no setor industrial e no setor de varejo, a oferta de empregos em escritórios e o número de residências. Esses modelos foram elaborados com o objetivo de desenvolver um novo modelo de previsão de viagens de caminhão para o Conselho Metropolitano de Baltimore.

Iding et al. (2002) elaboraram modelos de atração e produção de viagens de carga para diferentes setores industriais, considerando como variáveis independentes a área total e o número de empregados. Observa-se que apenas em 4 casos o coeficiente de determinação foi superior a 0,7 , predominando valores inferiores a 0,5 .

A Tabela 2-B apresenta um estudo realizado no estado de Indiana com o objetivo de desenvolver um banco de dados dos fluxos de entrada e saída de carga em uma zona de tráfego (condados do estado de Indiana). Para tanto, Back (1999) utilizou uma modelagem baseada em volume de carga, desagregada por 19 tipos de carga, e aplicou regressão linear simples e múltipla. Observa-se que na maioria dos casos $(78,9 \%$ das vezes) o coeficiente de determinação $\left(R^{2}\right)$ foi superior a 0,7 . Em todos os casos, o $R^{2}$ foi superior a 0,5 .

O estudo realizado por ITE (1995) apresenta modelos baseados em viagens de veículos e elaborados a partir de regressão linear simples. Como variáveis independentes, foram utilizadas o número de docas existentes nos terminais e o número de empregados.

A Tabela 2-C apresenta modelos baseados em viagens de veículos para cada tipo de categoria de viagens contempladas no estudo elaborado por Ogden (1977). O autor apresenta também modelos baseados em volume de carga que são aplicados a cada um dos sete tipos de carga considerados. Para ambas as aplicações, são utilizados modelos de regressão simples e múltipla. Analisando a Tabela 2-C, pode-se verificar que os modelos elaborados com base em viagens de veículos apresentam um maior coeficiente de determinação do que os modelos elaborados com base em volume de carga.

Hutchinson (1974) elaborou os modelos de geração de viagens com uma modelagem baseada em viagem de veículos e regressão linear simples. Pode-se observar que o modelo elaborado para a produção de viagens possui coeficiente de determinação com valor superior ao elaborado para a atração de viagens. Além desse estudo, apresentam-se também modelos de geração de viagens elaborados, considerando os setores de alimentos e bebidas, impressão e publicação, ma- quinaria e papel e produtos afins. Dos 8 modelos apresentados, apenas 2 apresentam coeficiente de determinação maior que $0,7(25 \%)$.

A Tabela 2-D apresenta um estudo realizado com o objetivo de identificar a geração de viagens por caminhão e obter dados para uma avaliação da situação observada na região em estudo (Tadi e Baldach, 1994). Os autores utilizam uma modelagem baseada em viagens de veículos e apresentam as taxas diárias de geração de viagens para caminhões com base na área do PGV Carga observado.

No estudo realizado por Ogden (1992), utilizou-se uma modelagem com base em viagens de veículos gerando taxas de viagens por caminhões e por tipo de uso do solo. Como variável independente, utilizou-se a área construída, medida em $92,9 \mathrm{~m}^{2}$.

A Tabela 2-E apresenta 9 modelos analisados por Iding et al. (2002). Dentre esses modelos, encontramse 3 trabalhos realizados nos Estados Unidos, que tinham como foco o transporte de carga entre estados, e 5 trabalhos realizados na Holanda, que tinham como objetivo principal a geração de viagens de carga em setores industriais e centros urbanos.

Christiansen (1979) realizou um estudo com o objetivo de estimar as viagens geradas para shoppings centers na região de Nova York. Na Tabela 2-E é possível observar o resultado desse estudo, em que se pode verificar que o autor utilizou uma modelagem baseada em viagens de veículos e apresenta as taxas de paradas diárias de caminhão, tendo como variável independente a área construída, medida em $929 \mathrm{~m}^{2}$.

Muñuzuri et al. (2009) realizou um estudo com foco no transporte de carga urbana entre atacadistas e varejistas, na cidade de Sevilla, na Espanha. Os autores buscaram desenvolver um modelo que melhor se adequasse à realidade urbana e que necessitasse de um menor número de dados para sua utilização, tornandose, assim, mais simples em sua aplicação. A modelagem escolhida se assemelha ao modelo gravitacional.

O estudo realizado por Nuzzolo et al. (2008), na Itália, teve por objetivo estimar os fluxos internacionais (importação/exportação) por rodovia. $\mathrm{O}$ autor utiliza uma modelagem baseada em volume de carga, desagregada por tipo (s) e por zona, para um dado período de tempo (h) e considera regressão múltipla.

Inicialmente, o autor apresenta uma formulação genérica em que a variável independente $(\mathrm{x})$ pode ser considerada como qualquer variável socioeconômica ou de nível de serviço e, posteriormente, um modelo utilizando as variáveis independentes: número de habitantes e empregados por zona, o número de empregados relacionados ao tipo de carga, rendimento médio per capita e PIB per capita. Além disso, o autor considera a localização da zona em estudo e se esta tem ou não porto. 


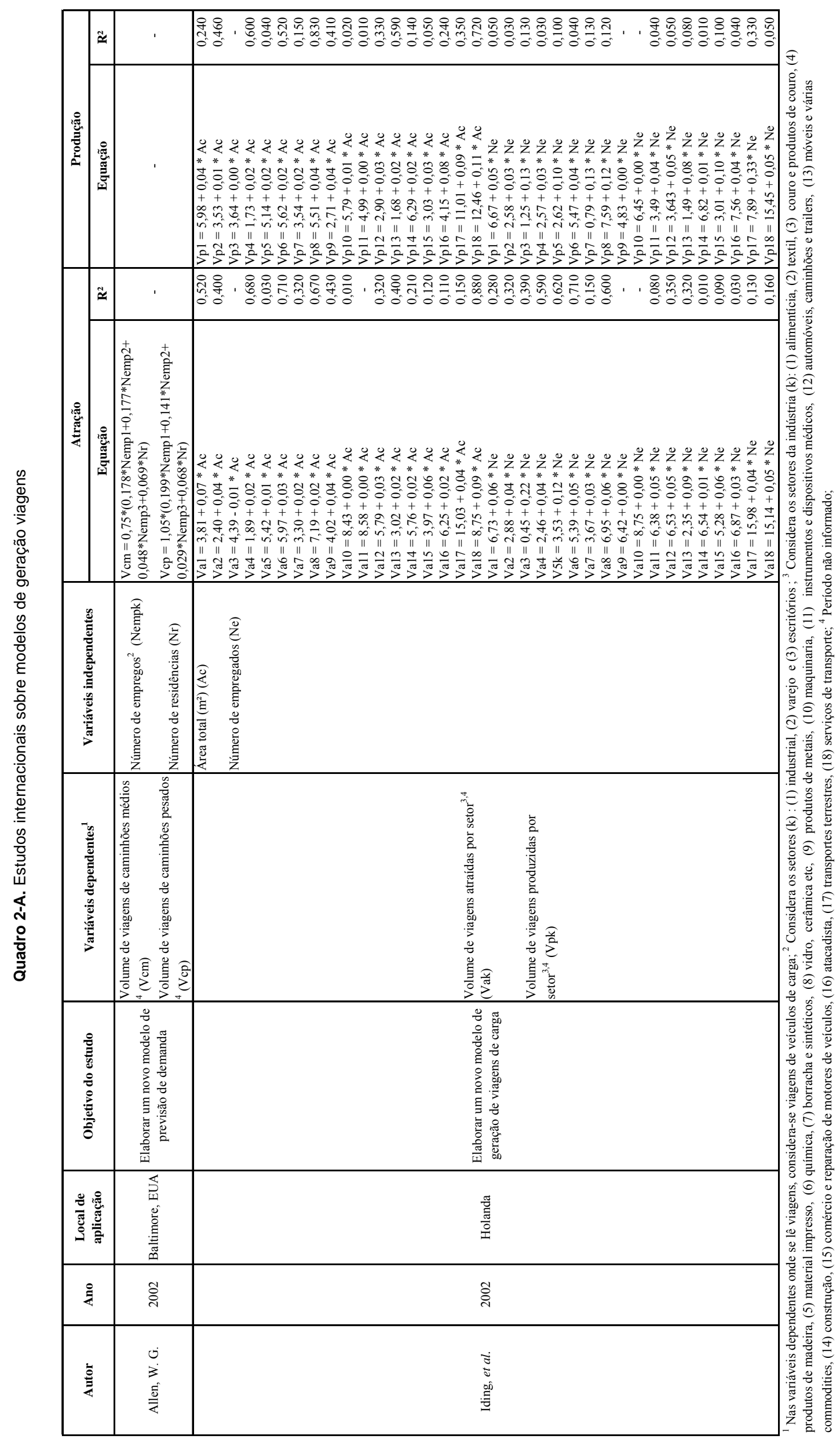




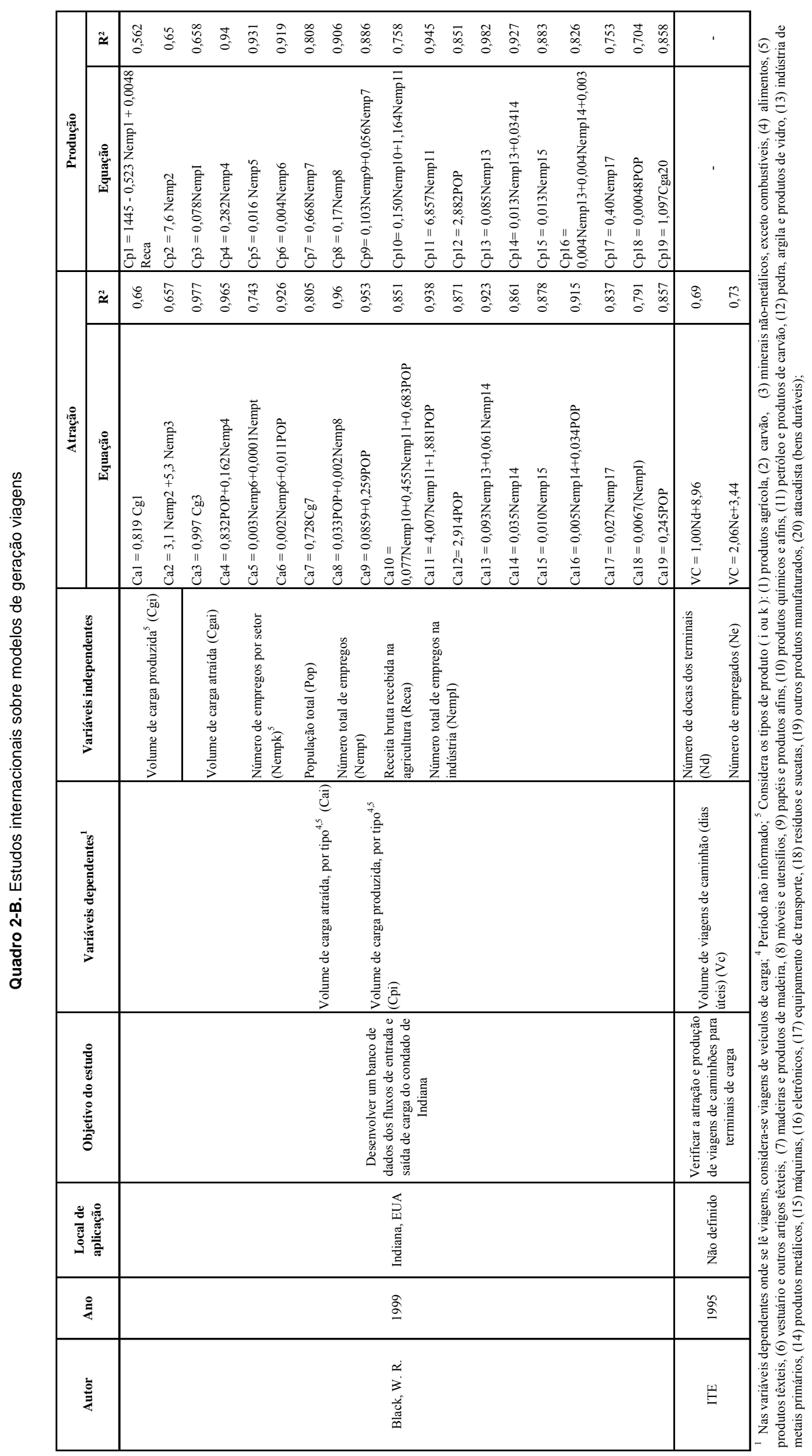




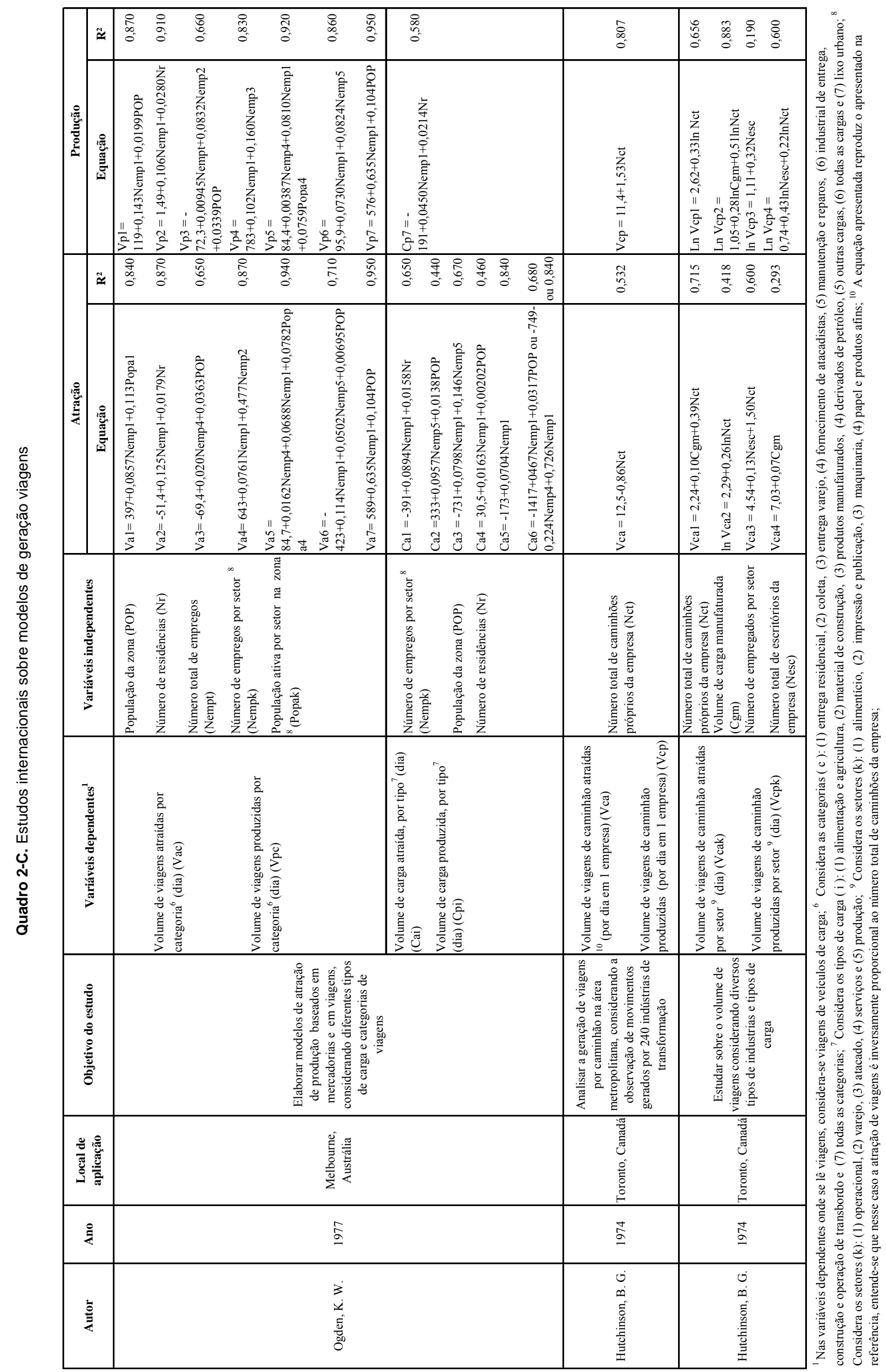




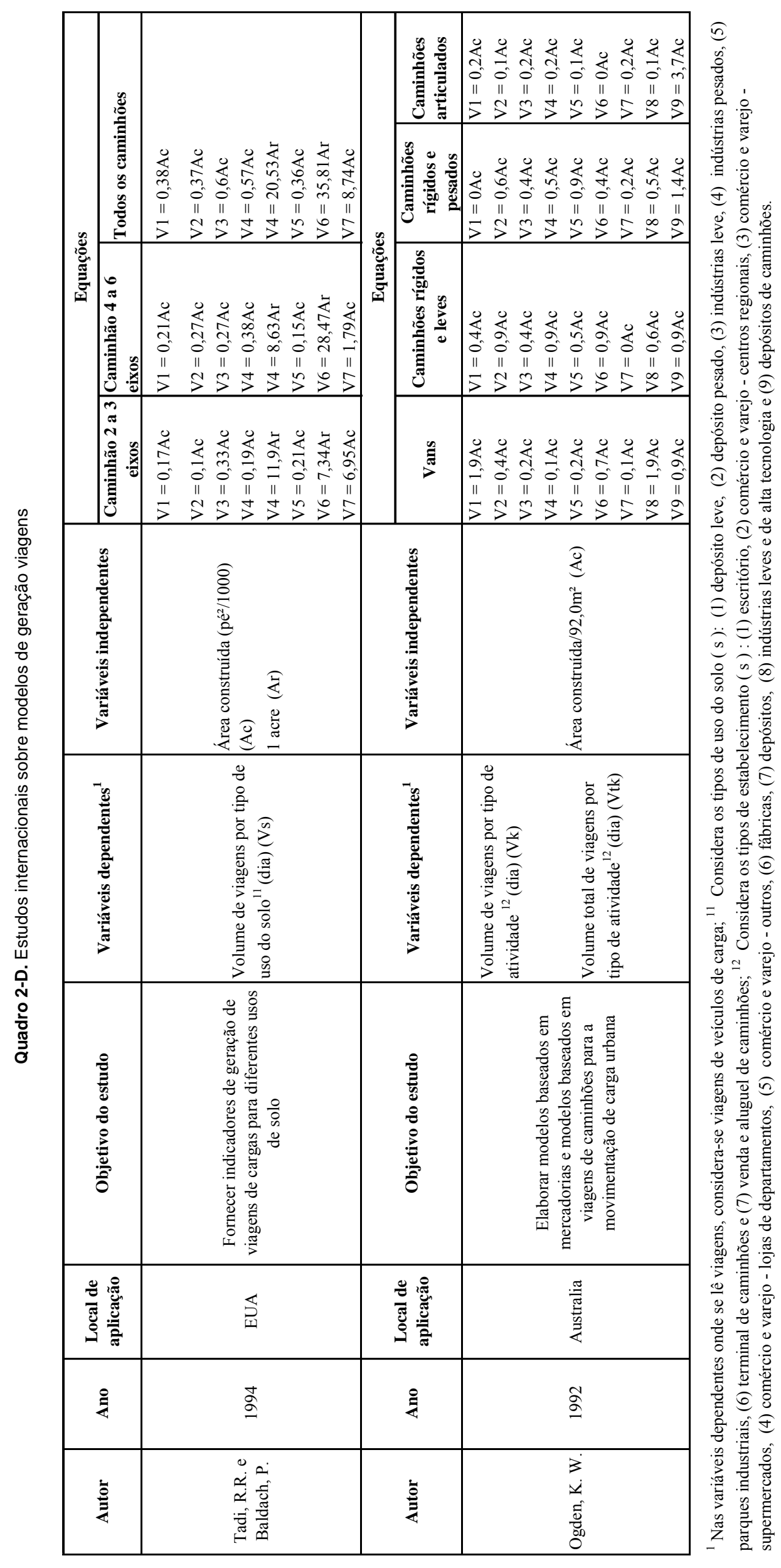




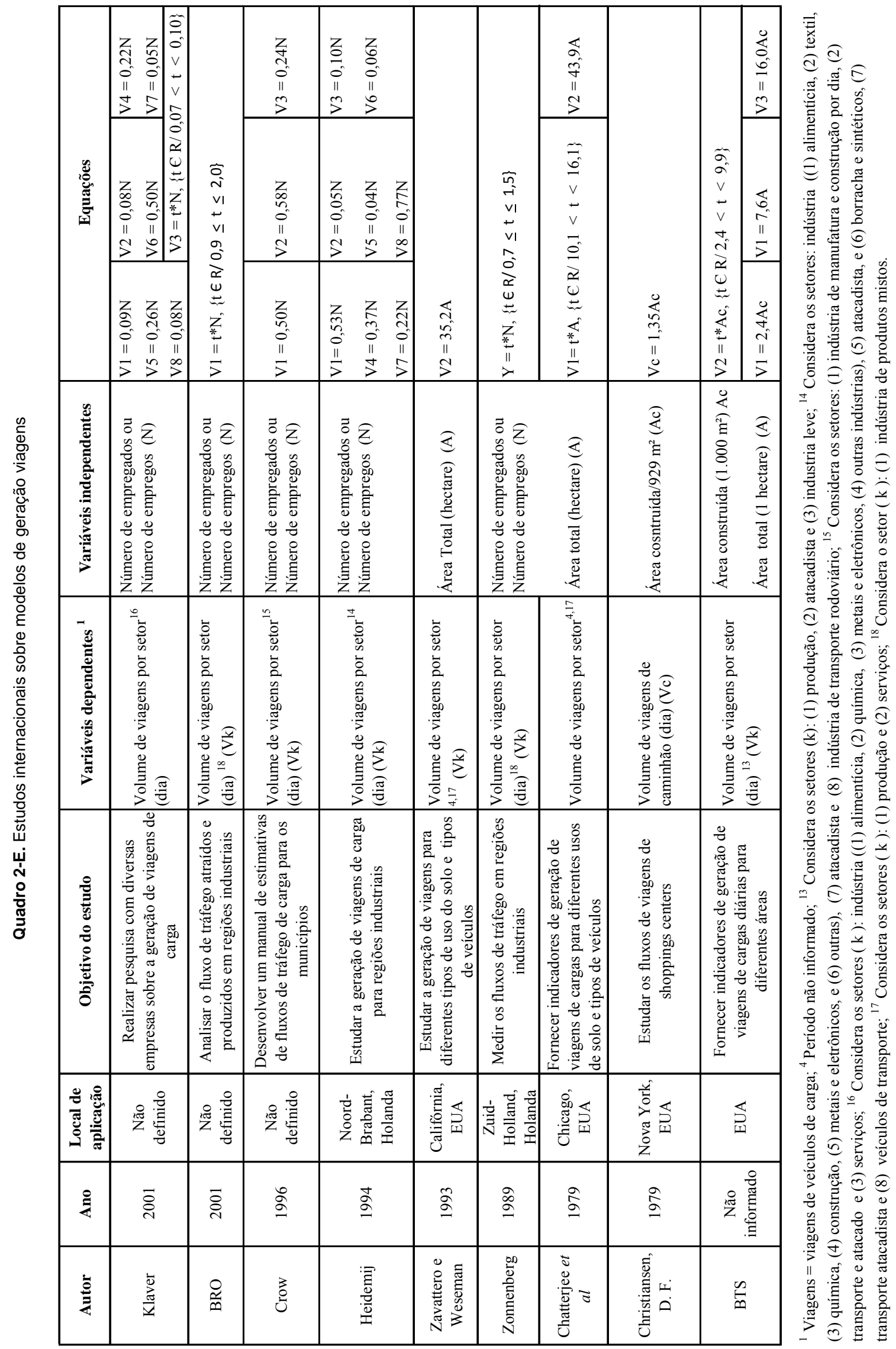


Morlok (1978) apresenta modelos que fazem uma relação entre a demanda de carga (atração e produção) e o tempo. Portanto, sua aplicação em estudos de PGV Carga deve considerar que o empreendimento já se encontra instalado e que se deseja determinar qual a evolução do tráfego de caminhões ou do fluxo de carga na medida em que o tempo passa. Para isso, é necessária a criação de um histórico do tráfego de caminhões ou do fluxo de carga. Como em toda projeção, deve-se tomar cuidado com o horizonte a ser considerado. Como se está fazendo uma previsão da demanda futura de viagens em função do seu comportamento no passado, esse tipo de modelo é incapaz de considerar variações não previstas ou imponderáveis.

A partir de Holguín-Veras e Thonson (2000), verificou-se que os modelos de geração de viagens utilizados em análise e previsão de demanda do transporte de carga podem ser baseados em deslocamento de carga (volume de carga) ou volume de viagens de veículos. Na revisão bibliográfica apresentada neste trabalho, verificou-se uma predominância no uso de modelos de geração de viagens que utilizam o volume de viagens como variável dependente. Dos 4 trabalhos nacionais considerados, 3 utilizam o volume de viagens como variável dependente e dos 19 trabalhos internacionais considerados, 18 utilizam o volume de viagens como variável dependente, sendo que no trabalho de Iding et al. (2002) a escolha não é clara. Essa situação vai ao encontro da afirmação de Ogden (1992) e de Holguín-Veras e Thonson (2000) quanto à facilidade de obtenção dos dados de tráfego, o que facilitaria a proposição de modelos de geração de viagens que empregam como variável dependente o volume de viagens de caminhão.

$\mathrm{Na}$ literatura consultada nem sempre é clara a forma de determinar o volume de viagens de caminhão que são produzidas ou atraídas para uma região. No âmbito nacional, no trabalho de Gasparini (2008) houve a preocupação de obter uma classificação do número de viagens por tipo de veículo de carga. Entretanto, a proposição dos modelos de geração de viagens se divide em geração de viagens de veículos de carga (todos os tipos) e de caminhões. Na literatura internacional, Allen (2002) divide seus modelos para caminhões médios e pesados, Tadi e Baldach (1994) dividem seus modelos por tamanho de veículo associado ao número de eixos e Ogden (1992) divide seus modelos por tipo de caminhão em rígidos leves e pesados e articulados.

Adicionalmente, Mello (2002), nas referências nacionais, e Iding et al. (2002), Tadi e Baldach (1994), Black (1999), Ogden (1992), Ogden (1977) e Hutchinson (1974), nas referências internacionais, dividem seus modelos por setor de atividade. Essa é uma característica específica do transporte de carga, em que a tipologia da carga e do setor de atividade ao qual o transporte esteja associado é determinante do perfil das viagens, o que obriga a elaboração de uma modelagem desagregada para a previsão de demanda.

Nas referências nacionais consideradas, predominam os modelos que utilizam regressão linear simples ou múltipla. Esses modelos também são os que apresentaram os melhores coeficientes de determinação. Como foi possível verificar na experiência nacional, em particular no trabalho de Marra (1999), modelos que consideram ajustes de curva para função potência $\left(y=a^{x}\right)$ ou polinomial $\left(y=a+b x+c x^{2}+d x^{3}+\ldots m x^{m}\right)$, não incluídos na Tabela 1 , não apresentaram resultado tão bom quanto aqueles que utilizaram regressão linear simples ou ajuste exponencial (incluídos na Tabela 1). Nesses casos, entende-se que a adequada escolha de uma variável independente possa contribuir mais para um bom resultado do modelo do que o uso de modelos excessivamente elaborados ou que utilizam muitas variáveis independentes.

Ao se observar as referências internacionais, a modelagem apresentada é diversificada, apresentando relações que consideram ajuste de curvas (regressão linear e polinomial), ajuste exponencial ou logarítmico e, a partir de uma abordagem simplificada, o estabelecimento de taxas. Nesse caso, quando se trabalha com ajuste de curvas, predomina o uso de regressão linear. Acredita-se que isso se deva a simplicidade de aplicação e análise dos resultados (análise de sensibilidade). A regressão linear pode ser simples ou múltipla, sendo que ambas são bastante utilizadas.

Ainda quanto às referências internacionais, é possível observar o uso de conversão em base logarítmica para as variáveis independentes na elaboração de regressões lineares (simples ou múltiplas), o que pode representar um método a ser testado nos estudos nacionais considerados, em que predomina o uso de regressões simples.

De uma forma geral, regressões lineares, simples ou múltiplas parecem apresentar bons resultados $\left(\mathrm{R}^{2} \geq\right.$ $0,7)$ quanto aos coeficientes de determinação encontrados. Embora possam ser encontrados valores entre 0,1 e 0,3 , predominam valores superiores a 0,7 . Aparentemente essa é uma indicação de que é possível obter resultados satisfatórios com modelos simples, que utilizam poucas variáveis independentes (uma ou duas). Também se poderia considerar que a complexidade da função matemática associada ao modelo está em um segundo plano quando comparada com a compreensão dos mecanismos que levam à escolha das variáveis independentes e dependentes.

As variáveis dependentes que se procura determinar são os volumes de viagens de veículos (caminhões) ou de carga (toneladas de carga transportadas). Já as variáveis independentes variam bastante. Quando se traba- 
lha com taxas, predomina a relação entre viagens (volumes de viagens de veículos ou cargas) e as áreas dos empreendimentos (área total ou área construída). Quando se trabalha com regressão linear, pode ser considerada uma diversidade de variáveis como: volumes de viagens de veículos, volume de carga, número de empregados e número de empregos (relacionados a um setor produtivo), população (total ou da região em estudo), áreas (total e construída), clientes, número de docas, entre outras.

Uma característica da modelagem da geração de viagem para o transporte de carga e para sua aplicação ao PGV Carga está na necessidade de se estabelecer diferentes equações ou taxas para diferentes tipos de carga e classes de veículos. Essa característica está associada à tipologia da carga e usualmente não se encontra nos modelos que se destinam à geração de viagens de passageiros. Com isso, para um mesmo par de regiões, é possível ter tantas equações quantos forem os tipos de carga e classes de veículos, sendo essas equações ajustadas de forma diferente e com diferentes coeficientes de determinação. Essa situação demonstra a complexidade do problema de modelar a geração de viagens de carga.

\section{CONSIDERAÇÕES FINAIS E SUGESTÕES}

Pólos Geradores de Viagens de carga (PGV Carga) são empreendimentos com potencial de produzir e atrair viagens de veículos de carga, como caminhões, camionetas, caminhonetes e furgões. O impacto desses empreendimentos no sistema viário quanto ao uso e ocupação do solo é diferenciado, dependendo da região e do porte do empreendimento. Entretanto, os impactos tendem a ser mais expressivos em áreas urbanas, onde já se verifica trânsito intenso de veículos motorizados e adensamento populacional.

Neste sentido, ferramentas que auxiliem na identificação dos volumes de viagens de veículos de carga produzidos e atraídos pelos PGV Carga podem ser consideradas como instrumentos de valor para o gerenciamento da mobilidade na região do entorno desses empreendimentos.

Entende-se que o objetivo deste trabalho foi atingido ao apresentar o perfil dos modelos de geração de viagens de carga que podem vir a ser utilizados para subsidiar os estudos sobre PGV Carga com abrangência nacional e internacional. Adicionalmente, foi possível realizar uma análise desses modelos, indicando as práticas que poderiam servir de referência para futuros trabalhos.

A despeito dos esforços empreendidos até o momento, no levantamento das experiências nacionais $\mathrm{e}$ internacionais com a proposição de modelos de geração de viagens de carga, obteve-se um número bem maior de experiências internacionais do que nacionais, podendo indicar que este seja um campo de pesquisa ainda a ser explorado no Brasil. Nesse contexto, verifica-se a relevância deste trabalho por buscar orientar a pesquisa de um tema pouco consolidado na experiência nacional.

Embora considerando a abrangência geográfica e temporal apresentada no item 2 , não se pretendeu realizar uma revisão bibliográfica exaustiva sobre o tema, sugerindo-se esse tipo de pesquisa para trabalhos futuros, no intuito de confirmar as tendências indicadas.

\section{REFERÊNCIAS BIBLIOGRÁFICAS}

Allen, W. G. (2002) Development of Truck Models. Transportation Consultant, Mitchells, Virginia.

Black, W. R. (1999) Commodity Flow Modeling. Transportation Research Board / National Research Council. Number E-C011, September 1999.

Crainic, T. G. e G. Laporte (1997) Planning models for freight transportation. European Journal of Operation Research 97 (1997) 409438 .

Christiansen, D. F. (1979) Urban transportation planning for goods and services. Technical report, TTI - Texas Transportation Institute. Final Report for the Federal Highway Administration.

Facchini, D. (2006) Análise dos "GAPS" de percepção dos atores envolvidos no transporte urbano de carga em Porto Alegre. Dissertação de Mestrado. Universidade Federal do Rio Grande do Sul. RS.

Gasparini, A. (2008) Atratividade do transporte de carga para pólos geradores de viagem em áreas urbanas. Dissertação de Mestrado em Engenharia de Transportes, Instituto Militar de Engenharia, RJ.

Hoeguin-Veras, J e E. Thorso (2000) Trip length distributions in commodity-based and trip-based freight demand modeling- investigations of relationships. Transportation Research Board. National Academy Press, $\mathrm{N}^{\mathrm{o}} 1707$. Washington.

Iding, M.H.E.; W. J. Meester e L. A. Tavasszy (2002) Freight trip generation by firms. Paper for the 42nd European Congress of the Regional Science Association Dortmund.

ITE (1995) Truck terminal trip generation. Institute of Transportation Engineering. Technical report, Summary Report by ITE Technical Council Commitee $6 \mathrm{~A}-46$., Washington, DC.

Marra, C. (1999) Caracterização de demanda de movimentações urbanas de carga. Dissertação de Mestrado em Engenharia Civil, Universidade Estadual de Campinas, SP.

Mcnally, M. G. (2000) The four-step model. In: Handbook of Transport Modelling, Edited by D. A. Hensher and K. J. Button, Elsevier Science Ltd.

Melo, I. C. B. (2002) Avaliação da demanda por transporte de carga em áreas urbanas. Dissertação de Mestrado em Engenharia de Transportes, Instituto Militar de Engenharia, RJ.

Morlok, E. K. (1978) Introduction to transportation engineering and planning. McGraw-Hill, US.

Muñuzuri, J.; P. Cortés; L. Onieva e J. Guadix (2009) Modeling freight delivery flows: the missing link of urban transport analysis. Journal of Urban Planning and Development. Aceito em 06/03/09 UP 1943.0000011

Nuzzolo, A.; U. Crisalli. e A. Comi (2008) A demand model for international freight transport by Road. European transport research review. Volume 1, Number 1, 23-33.

Ogden, K.W. (1992) Urban Goods Movement: A Guide to policy and Planning. Ashgate Publishing Limited; London.

Ogden, K.W. (1977) Modeling urban freight generation. Traffic Engineering \& Control. Publicado em March 1977.

Portugal, L.S. e L. G. Goldner (2003) Estudo de Pólos Geradores de Tráfego e de seus Impactos nos Sistemas Viários e de Transporte. Editora Edgard Clucher Ltda. São Paulo/SP.

Silva, J. e M. R. Waisman (2007) Cargas Urbanas: Estudo Exploratório sobre a Geração de Viagens de Caminhões em Bares e Restaurantes, $16^{\circ}$ Congresso da ANTP, Maceió, AL. 